09

\title{
Influence of magnetic confinement on the yellow excitons in cuprous oxide subject to an electric field
}

\author{
(C) J. Heckötter ${ }^{1}$, D. Fröhlich ${ }^{1}$, M. Aßmann ${ }^{1}$, M. Bayer ${ }^{1,2, q}$ \\ ${ }^{1}$ Experimentelle Physik 2, Technische Universität Dortmund, \\ D-44221 Dortmund, Germany \\ ${ }^{2}$ Ioffe Institute, Russian Academy of Sciences, \\ St. Petersburg, Russia \\ I E-mail: manfred.bayer@tu-dortmund.de
}

We study the spectrum of the yellow exciton series in crossed electric and magnetic fields. The electric field, applied along the optical axis, tilts the Coulomb potential between electron and hole, so that at sufficiently high fields exciton dissociation becomes possible, roughly when the electric dipole interaction energy exceeds the binding energy of an exciton state with principal quantum number $n$. For an applied voltage of $U=20 \mathrm{~V}$ all excitons above $n=6$ are dissociated. Additional application of a magnetic field normal to the optical axis introduces magnetic confinement, due to which above a threshold field strength around $B=2.5 \mathrm{~T}$ the exciton lines re-emerge. The complex dispersion with increasing fields suggests quantum chaotic behavior in this crossed field configuration, so that the search for exceptional points may be promising.

\section{Acknowledgements}

We gratefully acknowledge the support of this project by the Deutsche Forschungsgemeinschaft in the frame of the ICRC TRR 160 (project A1) and the project AS 459/1-3. MB also acknowledges support by the RF Government Grant N 14.Z50.31.0021.

DOI: $10.21883 /$ FTT.2018.08.46247.11Gr

* Полный текст статьи опубликован в журнале „Physics of the Solid State“ (T. 60. Вып. 8). 